\title{
ORDERED VECTOR SPACES
}

\author{
M. HAUSNER AND J. G. WENDEL
}

1. Introduction. An ordered vector space is a vector space $V$ over the reals which is simply ordered under a relation $>$ satisfying:

(i) $x>0, \lambda$ real and positive, implies $\lambda x>0$;

(ii) $x>0, y>0$ implies $x+y>0$;

(iii) $x>y$ if and only if $x-y>0$.

Simple consequences of these assumptions are: $x>y$ implies $x+z$ $>y+z ; x>y$ implies $\lambda x>\lambda y$ for real positive scalars $\lambda ; x>0$ if and only if $0>-x$.

An important class of examples of such $V$ 's is due to R. Thrall; we shall call these spaces lexicographic function spaces (LFS), defining them as follows:

Let $T$ be any simply ordered set; let $f$ be any real-valued function on $T$ taking nonzero values on at most a well ordered subset of $T$. Let $V_{T}$ be the linear space of all such functions, under the usual operations of pointwise addition and scalar multiplication, and define $f>0$ to mean that $f\left(t_{0}\right)>0$ if $t_{0}$ is the first point of $T$ at which $f$ does not vanish. Clearly $V_{T}$ is an ordered vector space as defined above. What we shall show in the present note is that every $V$ is isomorphic to a subspace of a $V_{T}$.

2. Dominance and equivalence. A trivial but suggestive special case of $V_{T}$ is obtained when the set $T$ is taken to be a single point. Then it is clear that $V_{T}$ is order isomorphic to the real field. As will be shown later on, this example is characterized by the Archimedean property: if $0<x, 0<y$ then $\lambda x<y<\mu x$ for some positive real $\lambda, \mu$.

Returning to the general case let $V$ be any ordered vector space, and $V^{+}$its set of positive elements. It is convenient to have a notation to indicate failure of the Archimedean property, as follows. Let $x, y \in V^{+}$. If $\lambda x<y$ for all positive real $\lambda$, we say that $x$ is dominated by $y$ and write $x \ll y$, or $y \gg x$. Clearly the relation $\ll$ is nonreflexive, nonsymmetric, and transitive; and $x \ll y$ implies $x<y$.

For given $x, y \in V^{+}$, if neither of $x$ and $y$ dominates the other we say that $x$ and $y$ are equivalent, and write $x \sim y$. This relation is characterized by the existence of positive real $\lambda, \mu$ such that $\lambda x<y<\mu x$, and it follows that it is indeed an equivalence relation on $V^{+}$. We denote the class of elements of $V^{+}$equivalent to given $x$ by $[x]$.

Presented to the Society, June 21, 1952; received by the editors March 3, 1952. 
Now we observe that there is a natural ordering on the set of equivalence classes; we define $[x]<[y]$ to mean that $x \gg y$. This definition is easily justified by the observation that if $x \sim x^{\prime}, y \sim y^{\prime}$, and $x \gg y$, then $x^{\prime} \gg y^{\prime}$. Our notation may be somewhat confusing; however, $[x]<[y]$ is to be thought of as meaning, roughly " $[x]$ comes before, or is more important than, $[y]$." An expression of frequent occurrence in the sequel is $[x] \leqq[y]$; this means that either $x$ dominates or is equivalent to $y$-hence that $y$ does not dominate $x$.

In the case where $V$ is an LFS, say $V=V_{T}$, it is easy to discern the meanings of dominance and equivalence. In fact, $f_{1} \gg f_{2}$ means that $f_{1}$ fails to vanish before $f_{2}$ does. More precisely, if $t_{i}$ is the first $t$ for which $f_{i}(t) \neq 0$, then $t_{1}<t_{2}$. From this it follows that $f_{1} \sim f_{2}$ if and only if $t_{1}=t_{2}$, and that $\left[f_{1}\right]<\left[f_{2}\right]$ if and only if $t_{1}<t_{2}$. In other words, the ordered set of equivalence classes is order isomorphic to the underlying set $T$.

Lemma 2.1. If $x \gg x_{1}, x_{2}, \cdots, x_{n}$ and $\lambda, \lambda_{1}, \lambda_{2}, \cdots, \lambda_{n}$ are positive real numbers, then

$$
\lambda x+\lambda_{1} x_{1}+\lambda_{2} x_{2}+\cdots+\lambda_{k} x_{k} \gg \lambda_{k+1} x_{k+1}+\cdots+\lambda_{n} x_{n} .
$$

Proof. We have $x>\left(n \mu \lambda_{i} / \lambda\right) x_{i}$ for all real $\mu>0$; therefore $(\lambda / n) x$ $>\mu \lambda_{i} x_{i}$, and $\lambda x>\mu\left(\lambda_{k+1} x_{k+1}+\cdots+\lambda_{n} x_{n}\right)$. Hence

$$
\lambda x+\lambda_{1} x_{1}+\lambda_{2} x_{2}+\cdots+\lambda_{k} x_{k}>\mu\left(\lambda_{k+1} x_{k+1}+\cdots+\lambda_{n} x_{n}\right),
$$

all positive real $\mu$, which is what we had to show.

Corollary 2.2. If $\left\{x_{t}\right\}$ is a set of elements of $V^{+}$no two of which are equivalent, then the $x_{t}$ are linearly independent.

Proof. If there is linear dependence among the $x_{t}$, we shall obtain an equation of the form

$$
\lambda x+\lambda_{1} x_{1}+\lambda_{2} x_{2}+\cdots+\lambda_{k} x_{k}=\lambda_{k+1} x_{k+1}+\cdots+\lambda_{n} x_{n},
$$

where all $\lambda$ 's are positive and real, all $x$ 's belong to the given set, and $x$ dominates $x_{1}, x_{2}, \cdots, x_{n}$. But, this, in view of Lemma 2.1 , is a contradiction.

Before stating the next lemmas it is convenient to introduce the notion of absolute value, defined by: $|x|=x,-x$, or 0 according as $x \in V^{+},-x \in V^{+}$, or $x=0$. Clearly the triangle inequality $|x+y|$ $\leqq|x|+|y|$ and the multiplicative relation $|\lambda x|=|\lambda||x|$ hold, for $x, y \in V$ and real $\lambda$.

LEMMA 2.3. If $[|x-y|] \leqq[|x-z|]$, then $[|x-y|] \leqq[|y-z|]$. 
Proof. We are given that $|x-z|$ does not dominate $|x-y|$, and must prove that $|y-z|$ does not dominate $|x-y|$. But if $|y-z|$ $>\lambda|x-y|$ for all real $\lambda$, then $\lambda|x-y|<|y-z| \leqq|x-y|+|x-z|$ for all $\lambda$, so that $(\lambda-1)|x-y|<|x-z|$ for all $\lambda$, which contradicts the assumption.

LEMMA 2.4. If $x \sim|y|$, there is a unique $\lambda$ such that $\lambda x=y$ or $|\lambda x-y|$ $\ll x$.

Proof. The uniqueness of $\lambda$ is immediate, for if $\lambda_{1} \neq \lambda_{2}$, we have $\left|\left(\lambda_{1}-\lambda_{2}\right) x\right| \leqq\left|\lambda_{1} x-y\right|+\left|\lambda_{2} x-y\right|$, and if both terms on the right were zero or dominated by $x$, we should have $x \ll x$.

To show that one such $\lambda$ exists we have $\mu x<|y|<\nu x$ for some positive real $\mu, \nu$. Let $\lambda^{\prime}$ be the supremum of the numbers $\mu$ for which $\mu x<|y|$. Then for $\epsilon>0$ we have $\left(\lambda^{\prime}-\epsilon\right) x<|y|<\left(\lambda^{\prime}+\epsilon\right) x$; therefore $-\epsilon x<|y|-\lambda^{\prime} x<\epsilon x$. Take $\lambda=\lambda^{\prime}$ or $-\lambda^{\prime}$ according as $y$ is positive or negative; changing signs if necessary we have $-\epsilon x<y-\lambda x<\epsilon x$. Therefore either $y-\lambda x=0$, or $|y-\lambda x| \ll x$; this completes the proof.

Sufficient machinery is now at hand for the investigation of structure questions. The finite-dimensional case is very easy; although the result is known $[1$, p. 240] we give it here as an illustration of the method.

TheOREM 2.5. Let $V$ be a finite-dimensional ordered vector space. $A$ basis $\left(e_{1}, e_{2}, \cdots, e_{n}\right)$ can be chosen so that the ordering in $V$ is lexicographic, i.e.,

$$
x=\sum_{i=1}^{n} \lambda_{i} e_{i}>0
$$

if and only if the first nonvanishing $\lambda_{i}$ is positive. In other words, $V$ is the lexicographic function space $V_{T}$ on the ordered set $T=(1,2, \cdots, n)$.

Proof. Let $V^{+}$be decomposed into equivalence classes as above, and for each equivalence class $t$ let $e_{t}$ be an arbitrary element of it; by Corollary 2.2 the set $\left\{e_{t}\right\}$ is finite. That is, $T=\{t\}$ is a finite set, and we may choose the notation so that $T=\{1,2, \cdots, k\}$ with $e_{1} \gg e_{2} \gg \cdots \gg e_{k}$; clearly $k$ does not exceed $n=\operatorname{dim} V$.

Let $y \in V$. If $y \neq 0$, then $|y|$ belongs to some equivalence class, say $|y| \sim e_{t_{1}}$. Applying Lemma 2.4 there is a unique $\lambda_{1}$ such that $\left|y-y_{1} e_{t_{1}}\right|$ $\ll e_{t_{1}}$, or $y=\lambda_{1} e_{t_{1}}$. We may now repeat the process on $y-\lambda_{1} e_{t_{1}}$, if it is not zero, and so on until the zero element is reached, as it must be in a finite number of steps. Thus we see that $y$ is indeed a linear combination of the $e_{t}$; since $y$ was arbitrary, it follows that the $e_{t}$ constitute a basis for $V$, and, moreover, that $k=n$. This completes the proof. 
CoRollary 2.6. If $V$ has the Archimedean property, then $\operatorname{dim} V=1$.

Proof. There is only one equivalence class.

It should be pointed out that there is a high degree of arbitrariness in the choice of basis for a finite-dimensional $V$. In fact, if $A$ is any lower triangular matrix with positive diagonal elements, then the equation $A e_{i}=e_{i}^{\prime}$ carries the basis $\left(e_{1}, e_{2}, \cdots, e_{n}\right)$ into another lexicographic basis $\left(e_{1}^{\prime}, e_{2}^{\prime}, \cdots, e_{n}^{\prime}\right)$. Conversely, any two bases are connected by a transformation of this form.

3. The embedding theorem for general $V$. It is evident that no such simple structure theorem will hold for arbitrary infinite-dimensional ordered vector spaces. For example, let $T$ be the set of positive integers in their natural ordering and form the lexicographic function space $V_{T}$. We get just the space of all real sequences, whose dimension as a vector space is the power of the continuum. But no vector space basis can be lexicographic in the sense of $\$ 2$, for the set of equivalence classes is in 1-1 correspondence with the points of $T$ and therefore is a countable set. A slight modification of this example shows that, moreover, not every $V$ is an LFS. Let $V$ be the subset of $V_{T}$ consisting of finite linear combinations of characteristic functions $f_{t}, t \in T$, where $f_{t}(s)=0$ or 1 according as $s$ differs from or equals $t$. The set of equivalence classes of $V$ is again isomorphic to $T$, so that if $V$ were an LFS, it would have to be isomorphic to $V_{T}$; but this is impossible since the dimension of $V$ is $\boldsymbol{\aleph}_{0}$.

The truth lies somewhere between these extremes; we shall show that associated with any $V$ there is a unique $V_{T}$ such that $V$ is isomorphic to a "large" subspace of $V_{T}$. Before stating the precise result we need a definition.

Let $V_{\boldsymbol{T}}$ be an LFS, let $t_{0} \in T$, and let $C$ be the linear transformation which truncates every $f \in V_{T}$ at $t_{0}$ - that is, $C f=g$, where $g(t)=f(t)$ for $t<t_{0}$ and $g(t)=0$ for $t \geqq t_{0}$. We shall call $C$ the $c u t$ determined by $t_{0}$.

Theorem 3.1. Let $V$ be an ordered vector space, let $T$ be the set of equivalence classes of $\mathrm{V}^{+}$, and for each $t \in T$ let a representative vector $e_{t} \in t$ be selected. Form the space $V_{T}$, denoting the characteristic function of the point $t$ by $f_{t}$. There is a mapping $F$ of $V$ to $V_{T}$ satisfying the following requirements:

(i) $F$ is linear;

(ii) $F$ is $1-1$;

(iii) $F$ is order preserving;

(iv) $F\left(e_{t}\right)=f_{t}, t \in T$;

(v) If $f \in F(V)$ and $C$ is any cut, then $C f \in F(V)$. 
This theorem has to be proved by a nonconstructive method. As a first step in the transfinite induction process we have:

THEOREM 3.2. Let $V_{0}$ be a proper subspace of $V$ which is mapped into $V_{T}$ by a function $F: y \rightarrow y^{\prime}$ satisfying (i)-(v) above. Let $x \notin V_{0}$, and let $V_{1}$ be the subspace spanned by $x$ and $V_{0}$. Then there is an extension of the mapping $F$ having domain $V_{1}$ and again satisfying (i)-(v). (We are assuming that (iv) is not vacuously satisfied; in other words that $V_{0}$ contains all of the $e_{t}$ )

Proof. Let $S$ be the set of equivalence classes $[|x-y|]$ for $y \in V_{0}$. We observe that $S$ has no last element. In fact, suppose that $[|x-y|]$ $\leqq[|x-z|]$ for some $z \in V_{0}$ and all $y$. Let $t$ be the equivalence class to which $|x-z|$ belongs; we have $|x-z| \sim e_{t}$, and therefore by Lemma 2.4 there is a constant $\lambda$ such that either $x-z=\lambda e_{t}$ or $\left|x-z-\lambda e_{t}\right| \ll e_{t}$. Since $z+\lambda e_{t}$ is again an element of $V_{0}$, both alternatives yield contradictions and we have the result.

Let $R$ be a well-ordered subset of $S$ which is cofinal in $S$, so that for $[|x-y|] \in S$ there is an $[|x-z|] \in R$ such that $[|x-y|]<[|x-z|]$. We index the elements of $R$ by ordinals $\alpha$ less than some limit ordinal $\theta$, obtaining $R=\left\{\left[\left|x-z_{\alpha}\right|\right]\right\}$, where $\alpha<\beta$ implies $\left[\left|x-z_{\alpha}\right|\right]$ $<\left[\left|x-z_{\beta}\right|\right]$. For each $\alpha<\theta$ let $t_{\alpha}$ denote the equivalence class $\left[\left|x-z_{\alpha}\right|\right] ;$ then $t_{\alpha}<t_{\beta}$ for $\alpha<\beta$.

From Lemma 2.3 it follows that $t_{\alpha}=\left[\left|x-z_{\alpha}\right|\right] \leqq\left[\left|z_{\alpha}-z_{\beta}\right|\right]$, and therefore $e_{t_{\alpha}}$ is not dominated by $\left|z_{\alpha}-z_{\beta}\right|$ for $\beta>\alpha$. Applying the mapping $F$ we find that $F\left(e_{t_{\alpha}}\right)=f_{t_{\alpha}}$ is not dominated by $\left|F\left(z_{\alpha}\right)-F\left(z_{\beta}\right)\right|$ $=\left|z_{\alpha}^{\prime}-z_{\beta}^{\prime}\right|$. Therefore $z_{\alpha}^{\prime}(t)-z_{\beta}^{\prime}(t)=0$ for $t<t_{\alpha}$. We can now define the function $x^{\prime}$ which is to be the image in $V_{T}$, under the extension of $F$, of the given element $x$. For any $t \in T$ which is less than some $t_{\alpha} \in R$ let $x^{\prime}(t)=z_{\alpha}^{\prime}(t)$, and for the remaining $t \in T$ set $x^{\prime}(t)=0$. This definition is legitimate, for if $t<t_{\alpha}$ and also $t<t_{\beta}$, then $z_{\alpha}^{\prime}(t)=z_{\beta}^{\prime}(t)$ and the function $x^{\prime}$ so defined clearly vanishes except at the points of a well-ordered set.

The mapping $F$ is now extended to all of $V_{1}=\left\{\lambda x+y \mid y \in V_{0}, \lambda\right.$ real $\}$ by defining $F(\lambda x+y)=\lambda x^{\prime}+y^{\prime}$; we shall verify that $F$ on $V_{1}$ has the properties (i) $-(\mathrm{v})$.

The requirements (i) and (iv) are immediately seen to hold. In order to prove (v) let $C$ be any cut, and let $f \in F\left(V_{1}\right)$. The element $f$ has the form $f=\lambda x^{\prime}+x y^{\prime}$, and therefore $C f=\lambda C x^{\prime}+C y^{\prime}$. If the $t_{0} \in T$ which determines $C$ is less than one of the $t_{\alpha}$, then $C x^{\prime}=C z_{\alpha}^{\prime}$ and $C f=C\left(\lambda z_{\alpha}^{\prime}+y^{\prime}\right)$ which by hypothesis is the cut of some element of $V_{0}$. If $t_{0}$ exceeds all $t_{\alpha}$, then $C x^{\prime}=x^{\prime}, C f=\lambda x^{\prime}+C y^{\prime}=\lambda x^{\prime}+y_{1}^{\prime}$ for some $y_{1} \in V_{0}$, so that $C f=F\left(\lambda x+y_{1}\right)$. 
We next show that the extension is $1-1$; it is enough to prove that $x^{\prime} \neq y^{\prime}$ for $y \in V_{0}$. Supposing the contrary let $y^{\prime}=x^{\prime}$ for some $y \in V_{0}$. Then $y^{\prime}(t)=z_{\alpha}^{\prime}(t)$ for $t<t_{\alpha}$, and therefore $\left|y^{\prime}-z_{\alpha}^{\prime}\right|$ does not dominate $f_{t_{\alpha}}$. Since $F$ preserves order on $V_{0}$, this implies that $\left|y-z_{\alpha}\right|$ does not dominate $e_{t_{\alpha}}$. Hence $t_{\alpha}=\left[\left|x-z_{\alpha}\right|\right] \leqq\left[\left|y-z_{\alpha}\right|\right]$. Applying Lemma 2.3 we have $\left[\left|x-z_{\alpha}\right|\right] \leqq[|x-y|]$ for all $\alpha$. But $R$ was cofinal in $S$ and $S$ has no last element; this contradiction yields the result. As a corollary to this we can state the following. Let $W$ be the set of $t$ at which $x^{\prime}$ does not vanish. Let $y \in V_{0}$ and let $Y$ be the set of $t$ at which $y^{\prime}$ does not vanish. If there are any $t \in Y$ which exceed all of the points of $W$, let $t_{0}$ be the least such. Then, it is not the case that $y^{\prime}(t)=x^{\prime}(t)$ for all $t<t_{0}$. For otherwise, let $C$ be the cut determined by $t_{0}$ and apply $C$ to $y^{\prime}$. We have $C y^{\prime}=x^{\prime}$, but $C y^{\prime}$ is the image of some $y_{1} \in V_{0}$.

Finally we have to show that the extension of $F$ preserves order. Let $x>y, y \in V_{0}$, and suppose that $x^{\prime}<y^{\prime}$. (We already know that $x^{\prime} \neq y^{\prime}$.) Let $t_{0}$ be the first point at which $x^{\prime}(t) \neq y^{\prime}(t)$. We have $x^{\prime}\left(t_{0}\right)$ $<y^{\prime}\left(t_{0}\right)$, and by the corollary just proved $t_{0}$ does not exceed all of the points of $W$. Hence there is a $t_{\alpha} \in R$ such that $t_{0}<t_{\alpha} . x^{\prime}\left(t_{0}\right)=z_{\alpha}^{\prime}\left(t_{0}\right)$ $<y^{\prime}\left(t_{0}\right)$, but $z_{\alpha}^{\prime}(t)=y^{\prime}(t)$ for $t<t_{0}$. Therefore $z_{\alpha}^{\prime}<y^{\prime}$. Since $F$ on $V_{0}$ is order preserving we have $z_{\alpha}<y$. Then $x>y>z_{\alpha}$, so that $y-z_{\alpha}<x-z_{\alpha}$ and $x-z_{\alpha}$ is not dominated by $y-z_{\alpha}$. Therefore $t_{\alpha}=\left[\left|x-z_{\alpha}\right|\right]$ $\leqq\left[\left|y-z_{\alpha}\right|\right]$. But then $y^{\prime}(t)=z_{\alpha}^{\prime}(t)$ for $t<t_{\alpha}$, and in particular for $t=t_{0}$.

This contradiction shows that $x>y$ implies $x^{\prime}>y^{\prime}$. In a similar way we can show that $x<y$ implies $x^{\prime}<y^{\prime}$. Therefore, if $\lambda x+y>0$ with $\lambda>0$ we have $x>-y / \lambda, x^{\prime}>-y^{\prime} / \lambda$, and so $\lambda x^{\prime}+y^{\prime}>0$; a similar calculation yields the result for negative $\lambda$. This completes the proof of (iii) and of the theorem.

Proof of Theorem 3.1. F's satisfying the hypotheses of Theorem 3.2 surely exist, since we may take, for example, $V_{0}$ equal to the span of the $e_{t}$ and define $F$ to be the linear extension of the function defined by (iv), the $e_{t}$ being linearly independent by Corollary 2.2. We partially order the set of all such mappings by the definition $F_{1}<F_{2}$ if $F_{2}$ is a proper extension of $F_{1}$. Clearly the hypotheses of Zorn's lemma are fulfilled, and there is a maximal $F$. The domain of $F$ is all of $V$, for otherwise by Theorem $3.2 F$ has a proper extension. Q.E.D.

\section{REFERENCE}

1. Garrett Birkhoff, Lattice theory, Amer. Math. Soc. Colloquium Publications, vol. 25, rev. ed., New York, 1949.

The Rand Corporation 